\title{
Exercício em esteira não elevou a temperatura do casco de equinos
}

\author{
Exercise on treadmill did not enhance the temperature of the equine hoof
}

\section{Carolina Berkman ${ }^{\mathrm{I}}$ Raquel Mincarelli Albernaz $^{\mathrm{I}}$ Roberta Carvalho Basile $^{\mathrm{I}}$ José Corrêa de Lacerda-Neto ${ }^{\mathrm{II}}$ Antonio de Queiroz-Neto ${ }^{\mathrm{I}}$ Guilherme de Camargo Ferraz ${ }^{\mathrm{I}}$}

\section{RESUMO}

\begin{abstract}
Objetivou-se avaliar em equino a influência do exercício progressivo e intenso realizado em esteira de alta velocidade sobre a temperatura de regiões da pele, incluindo face, pescoço e quartela, e do casco, incluindo coroa, parede e sola. Utilizaram-se cinco equinos Puro Sangue Árabes, desferrados, com idade média de $8 \pm 0,7$ anos, sendo duas fêmeas e três machos, com peso corpóreo médio de $420 \pm 10 \mathrm{~kg}$. A intensidade de esforço foi determinada por meio da quantificação do lactato sanguíneo. As temperaturas foram determinadas com o auxílio de termógrafo, antes, durante e após o exercício. Realizou-se análise de variância seguida pelo teste de Tukey, sendo $P \leq 0,05$. A lactacidemia aumentou, principalmente nas velocidades intensas. Houve redução das temperaturas das regiões da face, pescoço e quartela após o exercício e diminuição das temperaturas da parede, coroa e sola do casco durante o exercício, enquanto a temperatura da manta de rolamento da esteira não se alterou em nenhum momento. Constatou-se indiretamente que a perfusão sanguínea do casco diminuiu durante o teste de esforço tanto em sua fase aeróbia como na anaeróbia. Nenhuma das intensidades de esforço foi capaz de alterar a temperatura da manta de rolamento da esteira. A temperatura do casco diminuída como decorrência fisiológica do esforço físico imposto, retornou aos valores normais após o término do exercício.
\end{abstract}

Palavras-chave: cavalo, exercício, manta de rolamento, esteira, termografia.

\author{
ABSTRACT \\ The objective of the study was to evaluate the \\ influence of intense and progressive exercise performed on a
}

\begin{abstract}
high speed treadmill on the temperature of the skin of the face, neck, pastern, and hoof, including the crown, sole and wall. Five unshod Arabian horses were used, with a mean age of $8 \pm 0.7$ years, two females and three males, average body weight of $420 \pm 10 \mathrm{~kg}$. The exercise intensity was determined by quantification of blood lactate concentrations. Temperatures were determined with thermography before, during and after exercise. The effect of exercise on the temperature of the anatomic structures studied was evaluated by ANOVA for repeated measures followed by Tukey test, with $P \leq 0.05$. Blood lactate increased, especially at more intense speeds. There were decreases in temperature of the regions of the face, neck and pastern after the exercise, of the wall, crown and sole of the hoof during the effort. The temperature of the moving belt blanket bearing did not change at any time throughout the test exercise. It was found, indirectly, that the blood perfusion in the hoof decreased both during the aerobic and anaerobic phase of the exercise test. None of the effort intensities was able to change the temperature of treadmill moving belt. The temperature of the hoof reduced as a physiologic consequence of the exercise and returned to normal values after the end of the test.
\end{abstract}

Key words: horse, exercise, moving belt treadmill, thermography.

\section{INTRODUÇÃO}

Atualmente, muitas modalidades esportivas equestres utilizam equipamentos para avaliar e incrementar a qualidade do treinamento dos cavalos atletas, sendo que a esteira de alto desempenho tem sido empregada em muitas propriedades e centros de

'Laboratório de Farmacologia e Fisiologia do Exercício Equino (LAFEQ), Departamento de Morfologia e Fisiologia Animal (DMFA), Faculdade de Ciências Agrárias e Veterinárias (FCAV), UNESP - Universidade Estadual Paulista, Campus de Jaboticabal, 14884-900, Jaboticabal, SP, Brasil. E-mail: gferraz@fcav.unesp.br. *Autor para correspondência.

"Departamento de Cirurgia e Clínica Animal (DCCV), FCAV, UNESP, Jaboticabal, SP, Brasil. 
treinamento com esta finalidade (ERICKSON, 2006). Nos últimos anos, existe a clara tendência de intensificação do emprego desse equipamento tanto nas universidades como em centros avançados de treinamento.

Na esfera acadêmica, as esteiras são muito empregadas em variados tipos de pesquisa, incluindo o estudo da taxa metabólica de cavalos atletas durante o exercício (EATON et al., 1995), treinamento de grupamentos musculares específicos (ROBERT et al., 2000), além de serem fundamentais na realização de testes de esforço, já que proporcionam velocidades e intensidades precisas e protocolos fielmente replicáveis (FERRAZ et al., 2010).

De maneira geral, a atividade física produz energia térmica suficiente para elevar a temperatura corpórea em até $5^{\circ} \mathrm{C}$, sendo que este calor é difundido pelo sangue para a pele e trato respiratório para sua dissipação por meio da sudação e evaporação (JONES et al., 2006; HINCHCLIFF \& GEOR, 2008). Estudos sobre os possíveis efeitos da dissipação de calor produzido pela esteira durante o exercício, assim como a influência do atrito da manta de rolamento sobre as estruturas do casco são escassos. Segundo BOFFI (2006), o atrito da borracha que constitui a manta de rolamento produz aquecimento do casco, sendo recomendada a utilização de ferraduras pelos cavalos que realizam exercício físico na esteira.

Não obstante, as informações na literatura enfocam o efeito das condições ambientais internas dos laboratórios de fisiologia do exercício sobre a temperatura corpórea por meio de termômetros infravermelhos (PALMER, 1983) e de sensores acoplados anatomicamente com resina (MOGG \& POLLITT, 1992).

Alternativamente, a termografia tem sido empregada na Medicina e na Medicina Veterinária como método auxiliar de diagnóstico e no acompanhamento da evolução de terapias de inúmeras enfermidades, já que a dissipação do calor corpóreo se faz em grande parte por ondas eletromagnéticas de frequência infravermelha (EDDY et al., 2001), dependentes do fluxo sanguíneo subcutâneo proveniente da atividade metabólica tecidual, oriunda de processos inflamatórios, infecciosos, reumáticos ou traumáticos. Processos em que ocorrem vasodilatação e aumento da emissividade de radiação infravermelha, capturada pelo termógrafo (ANDRADE FILHO, 1999).

Isto posto, o propósito deste estudo foi demonstrar, por meio da técnica termográfica, possíveis variações térmicas na manta de rolamento da esteira e sua influência sobre regiões cutâneas e estruturas do casco de equinos submetidos a teste de esforço incremental precedido por uma fase de galope intenso.

\section{MATERIAL E MÉTODOS}

Utilizaram-se cinco equinos da raça PuroSangue Árabe (PSA), desferrados, com idade média de $8 \pm 0,7$ anos, sendo duas fêmeas e três machos com peso corpóreo médio 420,0 $\pm 19,0 \mathrm{~kg}$. Doze horas antes do procedimento experimental, os animais foram submetidos a um banho com água e sabão, com a finalidade de minimizar a influência de sujidades sobre a técnica termográfica, depois foram mantidos em piquete de coast-cross (Cynodon sp.), com sal mineralizado e água ad libitum. O concentrado da dieta (Omolene, Purina, Nestlé) foi fornecido na quantidade de $2 \mathrm{~kg}$ animal ${ }^{-1}$, duas vezes ao dia.

Imediatamente antes do início do exercício teste, os cavalos tiveram os cascos limpos sem utilização de água, evitando prejuízos sobre a técnica termográfica, por promover a dissipação momentânea do calor. Cada cavalo foi então submetido à aclimatação, sendo mantidos no ambiente de aferição por 20 minutos. Internamente, na sala climatizada, o ar condicionado foi desligado em cada momento de obtenção das imagens termográficas para evitar influência da corrente de ar. A temperatura e umidade do ar foram controladas integralmente com termohigrômetro digital portátil (HT-270 - Instrutherm - Itest Medição e Automação Ltda). Esse controle permitiu que a esteira $\left(\right.$ Galloper $^{\circledR} 5500$, Sahinco LTDA, Palmital, São Paulo, Brasil) e os equinos utilizados no presente estudo fossem submetidos ao mesmo ambiente experimental.

O primeiro momento para determinação das imagens termográficas foi antes do exercício. Seguiuse a fase de aquecimento e o teste de esforço. As aquisições das imagens termográficas foram obtidas dois minutos subsequentes ao término das etapas do teste com os cavalos parados e na posição quadrupedal. A figura 1 revela todas as etapas e os respectivos momentos de aquisição das imagens termográficas. Importante ressaltar que todas as imagens foram produzidas no mesmo ambiente laboratorial. Cada termografia foi realizada em até 15 segundos na seguinte ordem: face, pescoço, quartela, coroa, parede, sola e manta, respeitando sempre a distância de $20 \mathrm{~cm}$ do ponto escolhido, técnica preconizada por BASILE et al (2010a). Para mensuração da intensidade do esforço, determinada a partir da lactacidemia (YSI 2300 - Lactate Analyser. YSI Incorporated, EUA), coletaram-se amostras de sangue com tubos de pressão negativa contendo fluoreto de sódio, por meio de venopunção da jugular direita. Todas as coletas foram realizadas nos momentos descritos anteriormente. 


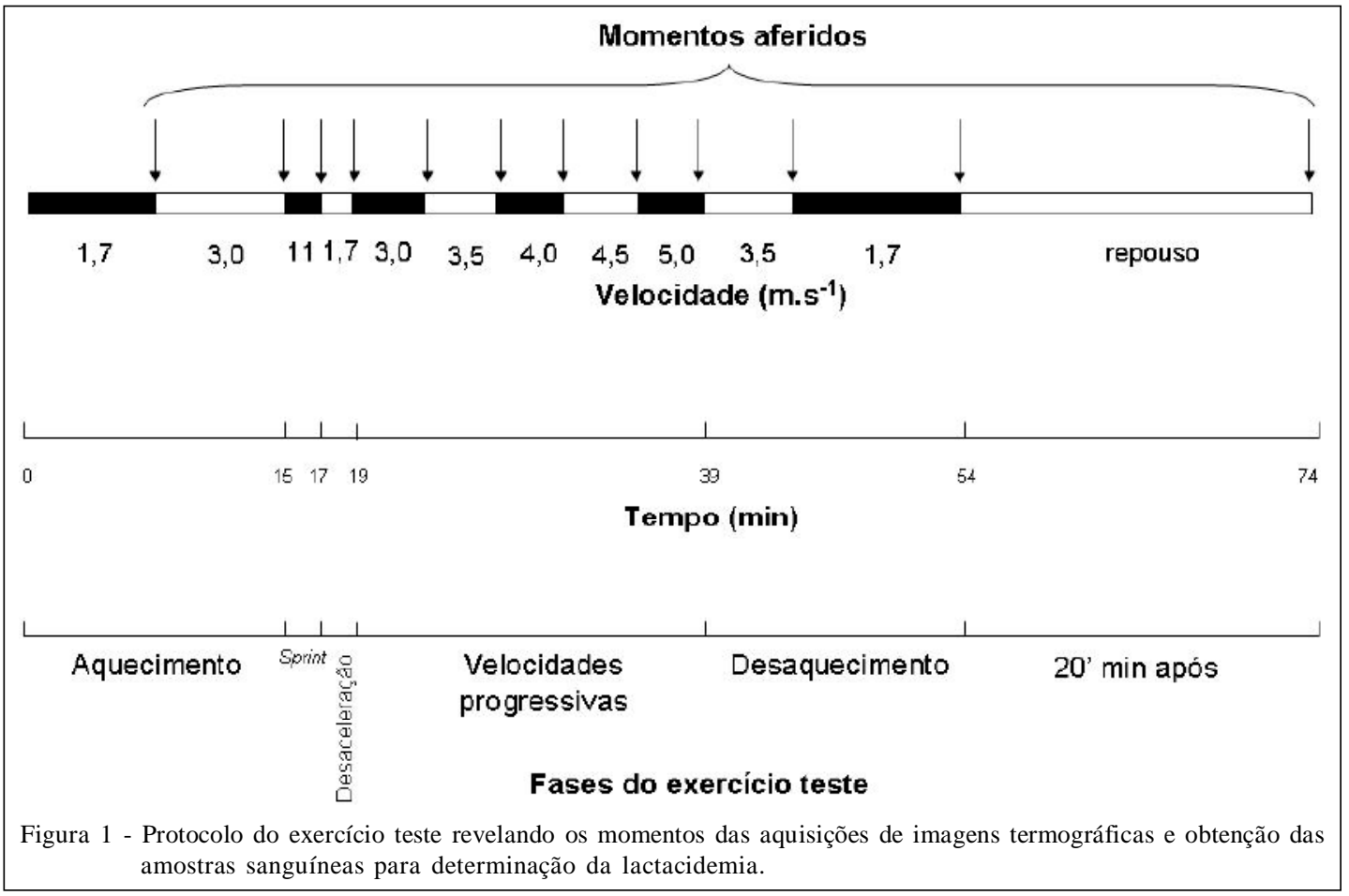

As temperaturas da pele da face, pescoço e quartela e do casco, sempre do antímero esquerdo, foram determinadas com o auxílio de termógrafo (FLIR $150^{\circledR}$ ) ajustado com emissividade de 0,98 (BASILE et al., 2010b). Na região facial, a avaliação termográfica foi realizada em um ponto central do músculo masseter. No pescoço, foi estabelecido ponto central entre o músculo rombóideo e o músculo esternocefálico. $\mathrm{Na}$ quartela, foi estabelecido um ponto no plano lateral no centro da região da falange média do membro torácico esquerdo. No casco, as regiões anatômicas avaliadas foram: muralha especificamente a região do plano axial dorsal, e a sola, no centro imediatamente anterior ao ápice da ranilha. Utilizaram-se as temperaturas máximas obtidas em cada localização anatômica. As temperaturas retais foram aferidas com termômetro clínico digital flexível (Incoterm ${ }^{\circledR}$ ) em todos os momentos.

Para verificação da normalidade dos dados (médias \pm desvio padrão) empregou-se o teste de Kolmogorov-Smirnov. O efeito do exercício sobre a temperatura das regiões anatômicas, da manta de rolamento da esteira e temperatura retal foi avaliado estatisticamente por meio de análise de variância (ANOVA) para medidas repetidas no tempo, seguido pelo teste de Tukey quando necessário. Sempre em relação à antes do exercício, as possíveis diferenças do esforço físico sobre a lactacidemia foram verificadas utilizando-se o teste t de student para amostras pareadas. Para todos os testes estatísticos empregou-se $\mathrm{P} \leq 0,05$.

\section{RESULTADOS E DISCUSSÃO}

Os resultados obtidos neste estudo são inéditos e contribuirão com informações importantes sobre a utilização de esteiras de alta performance para cavalos. Para tanto, utilizou-se a técnica termográfica que possui boa aplicabilidade, pois representa um método rápido, eficaz, não invasivo, sendo capaz de detectar elevações ou reduções precisas de temperatura, uma grandeza física representativa do calor, de qualquer superfície (BASILE et al., 2010a).

No que diz respeito à acurácia do método termográfico, alguns requisitos na aquisição das imagens devem ser considerados e evitados (TURNER et al, 2001), como sujidades, presença de água ou qualquer outro fator que possa alterar a emissão de ondas infravermelhas pela superfície corpórea de interesse. Sabe-se que a temperatura ambiente influencia diretamente na temperatura dos membros dos equinos (MOGG \& POLLITT, 1992). Por esse motivo, no presente estudo, a temperatura ambiente foi mantida praticamente constante a $20,4 \pm 0,35^{\circ} \mathrm{C}$ com umidade relativa do ar em $73,1 \pm 2,48 \%$, sendo controladas por meio de termohigrômetro digital, aparelho portátil mantido em funcionamento durante todo o período experimental.

Os cavalos são capazes de manter sua temperatura interna, apesar da influência do ambiente, por meio de mecanismos termorregulatórios elaborados 
(MCCONAGHY, 1994). Contudo, durante o exercício, a taxa de calor produzido excede a capacidade de dissipação, fazendo com que a temperatura interna se eleve em até $1^{\circ} \mathrm{C}$ por minuto durante exercícios extenuantes (SALTIN et al., 1968; JONES et al., 2006). Obviamente que essa elevação da temperatura corpórea possui um limite, sendo que, no presente experimento, a temperatura retal dos cavalos teve aumento de $36,9 \pm 0,18^{\circ} \mathrm{C}$ para $40,1 \pm 0,07^{\circ} \mathrm{C}$, equivalente a um incremento de $8 \%(\mathrm{P} \leq 0,0001)$ ao longo do tempo em relação ao esforço realizado (Tabela 1).

Para a quantificação da intensidade de esforço realizada pelos cavalos durante os testes de esforço, faz-se necessário determinar a gênese da resposta fisiológica que depende essencialmente da velocidade, distância e duração do exercício. Segundo ROGERS et al. (2007), a determinação da intensidade de esforço, por meio de variáveis físicas e fisiológicas, é um critério determinista, não subjetivo, que serve de base para comparação com outros estudos. O exercício teste realizado neste estudo teve a distância percorrida total de 9.318 metros. Adicionalmente, este trabalho utilizou a lactacidemia para a determinação do esforço físico, que teve valores médios antes do exercício de $0,60 \pm 0,13 \mathrm{mmol} \mathrm{L}^{-1}$, elevando-se $(\mathrm{P}<0,001)$ ao final da fase de sprint para $6,12 \pm 2,50 \mathrm{mmol} \mathrm{L}^{-1} \mathrm{e}$ ao final da fase incremental atingindo $1,38 \pm 0,35 \mathrm{mmol} \mathrm{L}^{-1}$ de lactato. De acordo com ROBERGS (2001), quando a intensidade do exercício é elevada, há um aumento na produção de prótons $\left(\mathrm{H}^{+}\right)$e lactato, devido à contribuição parcial do metabolismo anaeróbio para produção de energia exigida pela contração muscular. Portanto, pôde-se demonstrar que o esforço realizado mobilizou tanto a via aeróbia quanto a anaeróbia para produção de ATP.

Do ponto de vista prático, a tabela 1 revela que, embora a fase de sprint tenha demandado contribuição parcial da glicólise anaeróbia para produção de energia, significando um esforço considerável, essa etapa não foi capaz de provocar o aumento da temperatura cutânea dos animais. Isso ocorreu, provavelmente, devido à continuidade relativamente curta desta fase do exercício teste, pois a produção de calor está diretamente relacionada à duração do esforço (HODGSON et al., 1994). Nesse sentido, na fase progressiva (incremental), que teve duração de 16 minutos, somente a área do pescoço teve a temperatura elevada em $4 \%(\mathrm{P} \leq 0,0001)$ ao final da última etapa do teste de esforço, sendo que os valores aumentaram de $35,1 \pm 0,68^{\circ} \mathrm{C}$ para $36,6 \pm 0,35^{\circ} \mathrm{C}$.

Após 20 minutos da realização do exercício teste, as temperaturas da face e pescoço tiveram, respectivamente, redução significativa de 9 e 5,6\%

Tabela 1 - Temperaturas $\left({ }^{\circ} \mathrm{C}\right)$ representadas pela média \pm desvio-padrão (DP) das diferentes regiões anatômicas cutâneas submetidas à termografia antes, durante e após 20 minutos do exercício teste.

\begin{tabular}{|c|c|c|c|c|c|c|c|c|c|c|c|c|}
\hline \multirow{2}{*}{ Variáveis } & \multirow{2}{*}{$\begin{array}{l}\text { A } \\
0\end{array}$} & \multirow{2}{*}{$\begin{array}{l}\mathrm{AQ} \\
15\end{array}$} & \multirow{2}{*}{$\begin{array}{r}\text { S } \\
17\end{array}$} & \multirow{2}{*}{$\begin{array}{l}\text { DSL } \\
19\end{array}$} & \multicolumn{5}{|c|}{----------------Exercício progressivo---------------- } & \multicolumn{2}{|c|}{-----DSQ----- } & \multirow{2}{*}{$\begin{array}{c}\text { Após 20' } \\
74\end{array}$} \\
\hline & & & & & 23 & 27 & 31 & 35 & 39 & 44 & 54 & \\
\hline \multirow{4}{*}{ 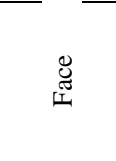 } & $\mathrm{ab}$ & $\mathrm{b}$ & $\mathrm{b}$ & $\mathrm{b}$ & $\mathrm{b}$ & $\mathrm{ab}$ & $\mathrm{b}$ & $\mathrm{b}$ & $\mathrm{a}$ & $\mathrm{ab}$ & $\mathrm{ab}$ & $\mathrm{c}$ \\
\hline & 33,4 & 32,8 & 32,9 & 32,8 & 33,6 & 34,0 & 34,2 & 34,4 & 34,6 & 34,3 & 33 & 30,4 \\
\hline & \pm & \pm & \pm & \pm & \pm & \pm & \pm & \pm & \pm & \pm & \pm & \pm \\
\hline & 1,11 & 0,40 & 0,52 & 0,66 & 0,63 & 0,42 & 0,40 & 0,66 & 0,53 & 0,78 & 1,27 & 1,28 \\
\hline \multirow{4}{*}{$\begin{array}{l}0 \\
0 \\
0 \\
0 \\
0 \\
2\end{array}$} & bcd & de & bcd & abcd & abcd & $a b c$ & $a b c$ & $a b$ & $\mathrm{a}$ & $a b c$ & cde & e \\
\hline & 35,1 & 34,5 & 35,2 & 35,5 & 35,7 & 36,0 & 36,2 & 36,3 & 36,6 & 36,0 & 34,9 & 33,7 \\
\hline & \pm & \pm & \pm & \pm & \pm & \pm & \pm & \pm & \pm & \pm & \pm & \pm \\
\hline & 0,68 & 0,81 & 0,65 & 0,34 & 0,33 & 0,36 & 0,38 & 0,44 & 0,35 & 0,43 & 1,17 & 0,63 \\
\hline \multirow{4}{*}{ 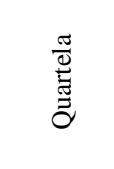 } & $a b$ & $a b$ & $a b$ & $a b$ & $\mathrm{a}$ & $\mathrm{a}$ & $a b$ & $\mathrm{a}$ & $\mathrm{a}$ & $\mathrm{a}$ & $a b$ & $\mathrm{~b}$ \\
\hline & 31,0 & 30,4 & 31,4 & 31 & 32,6 & 33,0 & 31,9 & 32,1 & 32,6 & 32,3 & 30,6 & 29,5 \\
\hline & \pm & \pm & \pm & \pm & \pm & \pm & \pm & \pm & \pm & \pm & \pm & \pm \\
\hline & 1,16 & 0,95 & 1,03 & 0,97 & 0,65 & 0,55 & 0,25 & 1,57 & 0,79 & 0,44 & 1,76 & 2,43 \\
\hline \multirow{4}{*}{$\begin{array}{l}\text { 芯 } \\
\cong\end{array}$} & $\mathrm{f}$ & ef & de & cde & bcd & bcd & $\mathrm{bc}$ & $\mathrm{bc}$ & $\mathrm{ab}$ & $\mathrm{a}$ & $\mathrm{a}$ & bcd \\
\hline & 36,9 & 37,4 & 37,8 & 38,3 & 38,6 & 38,6 & 38,9 & 39,1 & 39,2 & 40,1 & 40,0 & 38,5 \\
\hline & \pm & \pm & \pm & \pm & \pm & \pm & \pm & \pm & \pm & \pm & \pm & \pm \\
\hline & 0,18 & 0,19 & 0,15 & 0,39 & 0,39 & 0,44 & 0,4 & 0,45 & 0,38 & 0,07 & 0,51 & 0,75 \\
\hline
\end{tabular}

Médias seguidas de letras diferentes na mesma linha diferem entre si pelo teste de Tukey $(\mathrm{P}<0,05)$. A (antes do exercício); AQ (aquecimento); S (Sprint); DSL (desaceleração) e DSQ (desaquecimento).

Ciência Rural, v.41, n.8, ago, 2011. 
quando comparado à temperatura antes do exercício (Tabela 1). Essa diminuição, quando comparada com o momento antes do exercício, pode estar relacionada à atividade física que favorece a sudorese. Este fato, associado à umidade relativa do ar, favoreceu, segundo BICEGO et al. (2007), o processo de resfriamento evaporativo do suor, sendo este fenômeno a principal forma de se promover reduções na temperatura cutânea em mamíferos. Desta maneira, este mecanismo biológico pode ser explicado devido à manutenção praticamente constante da faixa de temperatura e umidade relativa do ar, que foram mantidas durante todo o experimento a $20^{\circ} \mathrm{C}$ e $73 \%$. Segundo WERNER (1998), nesta condição ambiental, a relação física entre a pressão cutânea de vapor d'água e a temperatura e umidade do laboratório permite que o resfriamento evaporativo ocorra.

Como a termografia possibilita localizar indiretamente alterações circulatórias provenientes de processos inflamatórios em membros de equinos (TURNER et al., 2001), foi possível detectar, no presente estudo, a dinâmica circulatória das estruturas do casco que apresentaram comportamento antagônico às estruturas cutâneas, principalmente em relação à região do pescoço, ou seja, tiveram as temperaturas diminuídas durante as etapas incrementais do exercício teste.

Com relação às estruturas do casco estudadas, houve clara tendência de redução da temperatura com normalização 20 minutos após o teste de esforço. A temperatura da coroa do casco (Figura 2A) diminuiu ( $\mathrm{P} \leq 0,0001)$ somente na fase de desaquecimento. A temperatura da parede do casco (Figura 2B) sofreu redução após o sprint, permanecendo em declínio até o final da etapa de desaquecimento, retornando aos valores registrados antes do início do teste após 20 minutos. Já a estrutura da sola do casco (Figura 2C) permaneceu sem alterações até o final do exercício teste. Esses resultados podem ser explicados pela correlação entre a temperatura e a perfusão sanguínea do casco (DYSON et al., 2001). Apesar do conceito clássico, que o exercício provoca aumento do débito cardíaco e vasodilatação muscular contribuindo para perfusão tecidual, parece que a circulação periférica do casco sofre vasoconstrição. De acordo com BAILEY et al. (2004), a microcirculação do casco de equinos possui significativa quantidade de receptores alfa-adrenérgicos. Esse fato pode explicar os resultados deste trabalho, pois o exercício estimula a liberação de catecolaminas, que sensibilizam receptores adrenérgicos $\alpha_{1}$ (FERRAZ et al., 2009) causando vasoconstrição, redução da perfusão e redução fisiológica da temperatura local.
No que concerne à temperatura da manta de rolamento da esteira, não foi observada qualquer alteração significativa ao longo do exercício teste. Sendo a temperatura inicial de $29,2 \pm 5,3^{\circ} \mathrm{C}$, ao final do sprint de $29,4 \pm 2,7^{\circ} \mathrm{C}$ e ao final da fase progressiva de $29,2 \pm 2,7^{\circ} \mathrm{C}$, comprovando que não ocorre qualquer alteração de temperatura desta superfície proveniente de atritos ou de qualquer outro agente mecânico intrínseco ao equipamento, contradizendo BOFFI (2006), que afirma a ocorrência de um superaquecimento da manta, tornando o uso deste equipamento deletério às estruturas do casco do equino quando desprovido de ferraduras.

Não houve alteração na temperatura da manta, que pode ser explicada pela reduzida força de atrito acumulada, que ocorre principalmente durante $o$ galope. Uma explicação plausível para esse evento é a menor geração de calor relacionado ao reduzido tempo de apoio que ocorre durante este tipo de andamento e consequente redução da força de atrito acumulada. Além disso, é importante enfatizar a ocorrência de perda de calor durante fases mais aceleradas pelo processo de convecção (ÖZISIK, 1985).

Cabe ressaltar ainda que a experiência do nosso laboratório indica que a utilização de ferraduras nos cavalos, durante os testes de esforço ou em sessões de condicionamento físico, reduz a vida útil da manta de rolamento. Além disso, o risco, apesar de pouco significativo, de uma ferradura se soltar do casco nos exercícios intensos deve ser considerado. Parece claro que o uso de ferraduras vai estar diretamente relacionado ao tipo de cavalo e manejo. A realidade mostra que essa abordagem deve ser diferenciada em cavalos de proprietários que nem sempre vão autorizar o desferrageamento dos seus animais. Os resultados encontrados no presente estudo são fortalecidos quando comparados a estudo abrangente (FRANKLIN et al., 2010) que investigou, em vários laboratórios de fisiologia do exercício equino, a incidência e tipo de injúrias associadas a esteiras de alta velocidade, confirmando a relativa segurança deste procedimento.

\section{CONCLUSÃO}

Os resultados obtidos neste estudo revelaram que exercício físico realizado em esteira não elevou a temperatura da manta de rolamento e que o casco sofreu redução fisiológica da temperatura, pois, ao término do esforço físico, esta retornou aos valores iniciais.

\section{COMITÊ DE ÉTICA E BIOSSEGURANÇA}

Protocolo de n.018031/09. 


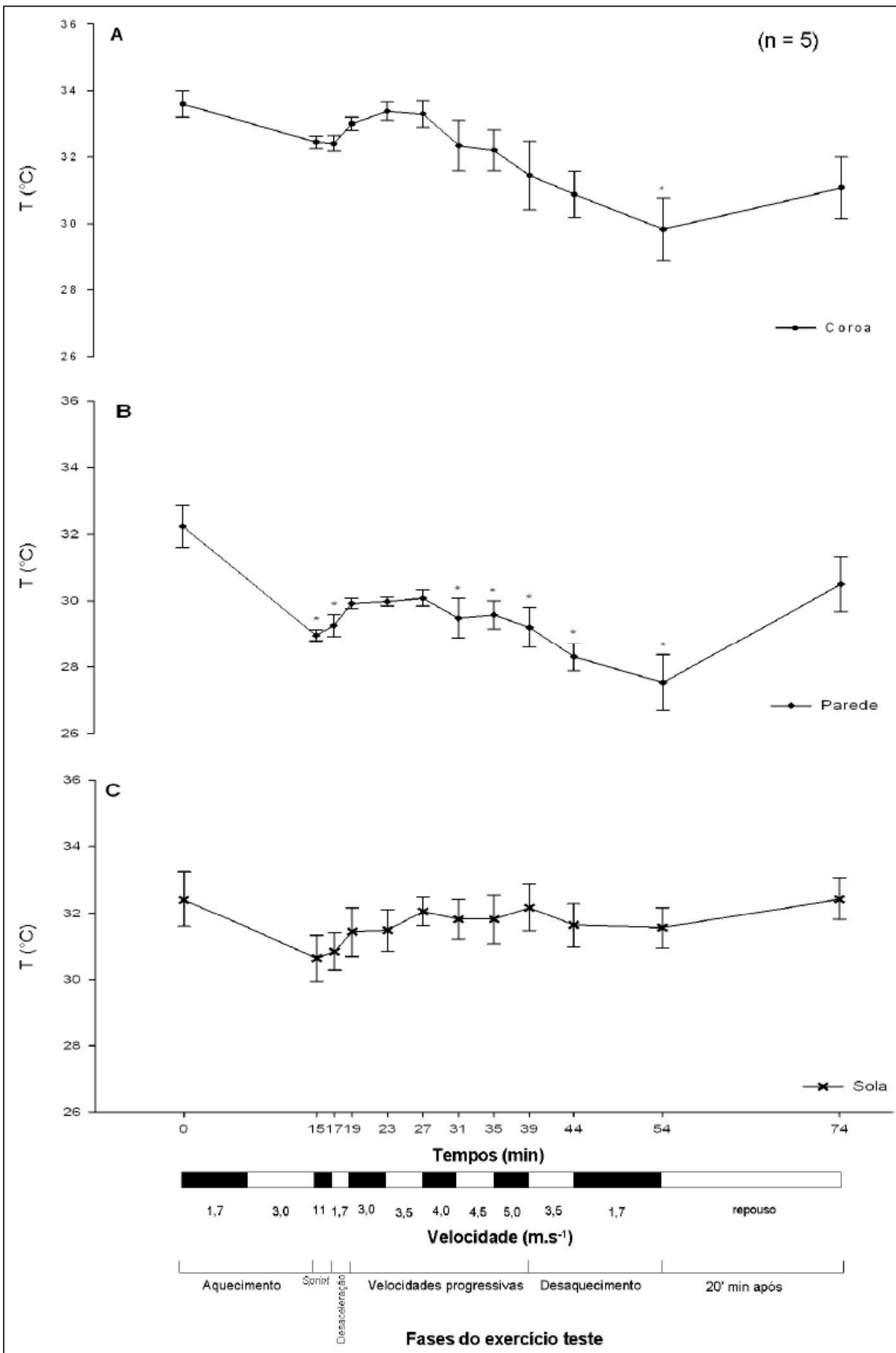

Figura 2 - Temperaturas $\left(\mathrm{T}^{\circ} \mathrm{C}\right)$ representadas pelas médias \pm desvio-padrão das diferentes estruturas do casco durante exercício teste em esteira. * Indica redução quando comparado com antes do teste de esforço.

\section{REFERÊNCIAS}

ANDRADE FILHO, A.C. Teletermografia: princípios físicos, fisiológicos e fisiopatológicos da produção da imagem e suas indicações na clínica de dor e reabilitação. Acta Fisiátrica, v.6, n.2, p.55-59, 1999. Disponível em: <http:// www.actafisiatrica.org.br>. Acesso em: 12 out. 2010.

BAILEY, S.R. et al. Current research and theories on the pathogenesis of acute laminitis in the horse. Veterinary 
Journal, v.167, n.2, p.129-142, 2004. Disponível em: <http:/ /www.sciencedirect.com>. Acesso em: 22 out. 2010. doi: 10.1016/S1090-0233(03)00120-5.

BASILE, R.C. et al. Guia prático de exames termográficos em equinos. Revista Brasileira de Medicina Equina, v.6, n.31, p.24-28, 2010a.

BASILE, R.C. et al. Equine inflammatory process evaluation using quantitative thermografic methodology. Ars Veterinária, v.26, p.77-81, 2010a. Disponível em: <www.arsveterinaria.org.br>. Acesso em: 22 nov. 2010 b.

BICEGO, K.C. et al. Physiology of temperature regulation: comparative aspects. Comparative Biochemistry Physiology Part A., v.147, p.616-639, 2007. Disponível em: <www.sciencedirect.com>. Acesso em: 01 set. 2010. doi: 10.1016/j.cbpa.2006.06.032

BOFFI, F.M. Principios de entrenamiento. In: BOFFI, F.M Fisiologia del ejercicio en equinos. Buenos Aires: InterMédica, 2006. Cap.16, p.223-241.

EATON, M.D. et al. Effect of treadmill incline and speed on metabolic rate during exercise in Thoroughbred horses. Journal of Applied Physiology, v.79, n.3, p.951-957, 1995. Disponível em: <http://jap.physiology.org>. Acesso em: 29 ago. 2010

ERICKSON, H.H. History of horse-whims, teamboats, treadwheels and treadmills. Equine Veterinary Journal Supplement, v.36. p.83-87, 2006. Disponível em: <www.sciencedirect.com>. Acesso em: 14 out. 2010. doi: $10.1053 / \mathrm{tvj} 1.2001 .0618$.

HODGSON, D.R. et al. Thermoregulation in the horse in response to exercise. British Veterinary Journal, v.150, n.3 p.219-235, 1994. Disponível em: www.sciencedirect.com. Acesso em: 12 set. 2010

FERRAZ, G.C. et al. Alterações hematológicas e cardíacas em cavalos Árabes submetidos ao teste de esforço crescente em esteira rolante. Brazilian Journal of Veterinary Research and Animal Science, v.46, n.6, p.431-437, 2009. Disponível em: <http://www.fumvet.com.br/novo/revista/46/n6/431437.pdf>. Acesso em: 25 nov. 2010.

FERRAZ, G.C. et al. Influência do treinamento aeróbio sobre o cortisol e glicose plasmáticos em equinos. Arquivo Brasileiro de Medicina Veterinária e Zootecnia, v.62, p.23-29, 2010. Disponível em: 〈www.scielo.br〉. Acesso em: 03 jan. 2011. doi: 10.1590/S0102-09352010000100003

FRANKLIN, S.H. et al. Investigation of the incidence and type of injuries associated with high-speed treadmill exercise testing. Equine Veterinary Journal, v.42, p.70-75, 2010. Disponível em: 〈http://onlinelibrary.wiley.com>. Acesso em: 14 dez. 2010. doi: 10.1111/j.2042-3306.2010.00234.x.

HINCHCLIFF, K.W.; GEOR, R.J. The horse as an athlete: a physiological overview. In: HINCHCLIFF, K.W. et al. Equine exercise physiology: the science of the exercise in the athletic horse. Philadelphia: Saunders, 2008. p.2-10.

JONES, J.H. et al. Energetic cost of locomotion on different equine treadmills. Equine Veterinary Journal Supplement, v.36, p.365-369, 2006. Disponível em: 〈http://onlinelibrary.wiley.com〉. Acesso em: 29 set. 2010. doi: 10.1111/j.2042-3306.2006.tb05570.x.

McCONAGHY, F. Thermoregulation. In.: HODGSON, D.R.; ROSE, R.J. Principles and practice of equine sports medicine - The athletic horse. Phildelphia: Saunders, 1994. p.181-202.

MOGG, K.C.; POLLITT, C.C. Hoof and distal limb surface temperature in the normal pony under constant and changing ambient temperatures. Equine Veterinary Journal, v.24, p.134-139, 1992. Disponível em: <http:/ /onlinelibrary.wiley.com>. Acesso em: 10 set. 2010. doi: 10.1111/j.2042-3306.1992.tb02798.x.

ÖZISIK, M.N. Heat transfer: a basic approach. New York: McGraw-Hill International, 1985. p.226-280.

PALMER, S.E. Effect of ambient temperature upon the surface temperature of the equine limb. American Journal of Veterinary Research, v.44, n.6, p.1098-1101, 1983. Disponível em: 〈http://www.ncbi.nlm.nih.gov>. Acesso em: 10 nov. 2010.

ROBERGS, R.A. Exercise-induced metabolic acidosis: where do the protons come from? Sportscience, v.5, n.2, p.1-20, 2001. Disponível em: <http://www.sportsci.org>. Acesso em: 22 set. 2010

ROBERT, C. et al. The effects of treadmill inclination and speed on the activity of two hindlimbs muscles in the trotting horse. Equine Veterinary Journal, v.32, p.321-317, 2000. Disponível em: 〈http://onlinelibrary.wiley.com>. Acesso em: 13 set. 2010 . doi: 10.2746/042516400777032246.

ROGERS, C.W. et al. Describing workload and scientific information on conditioning horses. Equine Comparative Exercise Phisiology, v.4, p.1-6, 2007. Disponível em: <http:/ /journals.cambridge.org>. Acesso em: 14 set. 2010. doi: 10.1017/ S1478061507727408.

SALTIN, B. et al. Muscle temperature during submaximal exercise in man. Journal of Applied Physiology, v.25, 679-688, 1968. Disponível em: 〈http://jap.physiology.org>. Acesso em: 22 out. 2010

TURNER, T.A. et al. Thermographic assessment of racing Thoroughbreds. In: ANNUAL CONVENTION OF AMERICAN ASSOCIATION OF EQUINE PRACTITIONERS, 47., 2001, San Diego, United States of North America. Proceedings. San Diego: AAEP, 2001. V.47, p.344-346. Disponível em: <http://www.ivis.org>. Acesso em: 29 set. 2010.

WERNER, J. Biophysics of heat exchange between body and environment. In: BLATTEIS, C.M. Physiology and pathophysiology of temperature regulation. London: World Scientific, 1998. Cap.3, p.25-45. 